$(\leq \mathrm{IIA} / \geq \mathrm{IIB})$. All patients were treated with chemotherapy. We used Cox models with the sporadic group as the reference to obtain hazard ratios (HR) with corresponding 95\% confidence intervals (CI). Since BRCA1/2 mutation carriers who received a DNA test after EOC diagnosis survived at least until this DNA test, which may result in survival bias, we also performed prospective analyses including only BRCA1/2-associated EOC patients with a DNA test result before EOC diagnosis $(n=82)$ and their matched sporadic controls.

Results The mean follow-up was 4.4 years (range 0.1-30.1). For the first 5 years after EOC diagnosis, the HRs for PFS $(0.85,95 \%$ CI $0.73-0.98)$ and OS $(0.58,95 \%$ CI $0.49-0.69)$ were in favor of the BRCA1/2 EOC patients. In the prospective analyses, survival benefit withstand for PFS (HR 0.66, 95\% CI 0.45-0.98), and - to a lesser extent - for OS (HR $0.69,95 \%$ CI $0.44-1.1$ ).

Conclusions For EOC patients treated with chemotherapy, we confirmed survival benefit for BRCA1/2 mutation carriers. This may indicate higher sensitivity to chemotherapy, both in first line treatment and in the recurrent setting.

\section{OP019/\#123 CLEAR CELL OVARIAN CANCER IN NATIVE ASIANS COMPARED TO US ASIANS - IS THERE A DIFFERENCE?}

1/ Tunnage*, ${ }^{2} \mathrm{D}$ Lewis, ${ }^{3} \mathrm{M}$ Caesar, ${ }^{4} \mathrm{C}-\mathrm{I}$ Liao, ${ }^{5} \mathrm{~A}$ Chan, ${ }^{5} \mathrm{D}$ Lee, ${ }^{5} \mathrm{~A}$ Rohatgi, ${ }^{6} \mathrm{~K}$ Darcy, ${ }^{6} \mathrm{C}$ Tian, ${ }^{3,7} \mathrm{~J}$ Chan. ${ }^{1}$ New York University, Gynecologic Oncology, New York, USA; ${ }^{2}$ MedStar Washington Hospital Center, Obstetrics and Gynecology, Washington, USA; ${ }^{3}$ California Pacific Medical Center Research Institute, Gynecologic Oncology, San Francisco, USA; ${ }^{4}$ Kaohsiung Veterans General Hospital, Obstetrics and Gynecology, Kaohsiung City, Taiwan; ${ }^{5} \mathrm{Palo}$ Alto Medical Foundation Research Intitute, Obstetrics and Gynecology, Palo Alto, USA; ${ }^{6}$ Gynecologic Cancer Center of Excellence, Obstetrics and Gynecology, Washington, USA; ${ }^{7}$ California Pacific Medical Center, Obstetrics and Gynecology, San Francisco, USA

10.1136/ijgc-2021-IGCS.36

Objectives To study the incidence and trends of clear cell ovary cancer (CCOC) in the US and Taiwan.

Methods Data were obtained from the United States Cancer Statistics (USCS) Public Use Databases and Taiwan Cancer Registry of Taiwan Health and Welfare Data Center (HWDC) from 2001 to 2017. SEER*Stat 8.3.9, Joinpoint regression program 4.8.0.1, and Excel were used to calculate the incidence and trends.

Results Compared to Whites, Asians have nearly five-fold higher incidence of CCOC (2.44 vs 0.49 per 100,000). Native Asians have a 1.8 fold higher incidence vs. US Asians (1.58 vs. 0.86 per 100,000$)$. The peak age range of CCOC diagnosis in Native Asians is younger compared to US women (5054 vs. 65-69 years). From 2001 to 2017, there was an Increase in CCOC in both NAs and US Asians at $6.2 \%$ per year vs $2.1 \%$ per year, respectively. In a projected model, by 2029, the incidence of CCOC in NAs will surpass that of serous cancer in US Whites.

Conclusions Over the last 17 years the rate of clear cell ovary cancer has increased for both Asians residing in Asia and the United States and may surpass other cell types in next decade. Further studies are needed to evaluate the genetic and environmental factors responsible for this disparity.

\section{OP020/\#524 HEATH CARE RESOURCE AND COST IMPLICATIONS OF INTEGRATION OF MOLECULAR CLASSIFICATION IN THE MANAGEMENT OF ENDOMETRIAL CANCER}

${ }^{1} \mathrm{G}$ Hanley*, ${ }^{2} \mathrm{E}$ Thompson, ${ }^{3} \mathrm{~S}$ Scott, ${ }^{4} \mathrm{M}$ Kinloch, ${ }^{5} \mathrm{~J}$ Irving, ${ }^{6} \mathrm{~S}$ Leung, ${ }^{7} \mathrm{~S}$ Salvador, ${ }^{8} \mathrm{~L}$ Helpman, ${ }^{9} \mathrm{D}$ Vicus, ${ }^{1} \mathrm{~A}$ Jamieson, ${ }^{10} \mathrm{~V}$ Samouëlian, ${ }^{11} \mathrm{~S}$ Kean, ${ }^{12} \mathrm{~S}$ offman, ${ }^{13} \mathrm{C}$ ParraHerran, ${ }^{14} \mathrm{~A}$ Talhouk, ${ }^{15} \mathrm{M}$ Plante, ${ }^{7} \mathrm{~W}$ Gotlieb, ${ }^{16} \mathrm{C}$ Gilks, ${ }^{1} \mathrm{~J}$ Kwon, ${ }^{1} \mathrm{~J}$ Mcalpine. ${ }^{1}$ University of British Columbia and BC Cancer, Gynecology, Division of Gynecologic Oncology, Vancouver, Canada; ${ }^{2}$ Molecular Oncology, University of British Columbia, Vancouver, Canada; ${ }^{3}$ Dalhousie University, Obstetrics and Gynaecology, Halifax, Canada; ${ }^{4}$ University of Saskatchewan, Pathology and Laboratory Medicine, Saskatoon, Canada; ${ }^{5}$ University of British Columbia, Royal Jubilee Hospital, Pathology and Laboratory Medicine, Victoria, Canada; ${ }^{6}$ University of British Columbia, Genetic Pathology Evaluation Centre, Vancouver, Canada; 'McGill University, Jewish General Hospital, Gynecology Oncology, Montreal, Canada; ${ }^{8}$ Cheba medical center, Gynecologic Oncology, Ramat Gan, Israel; ${ }^{9}$ Sunnybrook Health Sciences Centre, Gynecologic Oncology, Toronto, Canada; ${ }^{10}$ Gynecologic Oncology Service, CHUM, Université de Montréal, Department of Obstetrics and Gynecology, Montreal, Canada; ${ }^{11}$ University of Manitoba, Obstetrics and Gynecology, Gynecologic Oncology, Winnepeg, Canada; ${ }^{12}$ Dalhousie University, Pathology, Halifax, Canada; ${ }^{13}$ Brigham and Woman's Hospital, Pathology, Boston, USA; ${ }^{14}$ University of British Columbia, Obstetrics and Gynecology, Vancouver, Canada; ${ }^{15}$ Hotel Dieu de Quebec, Gynecology Oncology, Quebec, Canada; ${ }^{16}$ UBC, Vancouver General Hospital, Pathology and Laboratory Medicine, Vancouver, Canada

\subsection{6/ijgc-2021-IGCS.37}

Objectives Increasing incidence and morbidity of endometrial cancer (EC) has resulted in high systemic cost burdens associated with management. Molecular classification is now being integrated into routine pathologic reporting, providing objective biologically-relevant data to direct care. We assessed the cost implications of this integration in a modern cohort of women with ECs.

Methods 994 women diagnosed with EC across Canada (2016) underwent retrospective molecular classification enabling us to determine their eligibility for current molecular stratification trials and $2020 \mathrm{ESMO} / \mathrm{ESTRO} / \mathrm{ESP}$ risk group assignment and treatment recommendations. We then calculated cost differences based on molecular subtype/ProMisEdirected change in surgical management, adjuvant therapy, clinic visits, pathology testing, and genetic counseling.

Results Total costs saved, even after correcting for cost of molecular testing for all 994 individuals (\$450-575 CAD/test) were $\$ 570,744-\$ 747,042 \mathrm{CAD}$ or $\$ 574-\$ 752$ CAD per capita. If we test MMR and p53 IHC for all patients but perform POLE sequencing only in women where treatment would be altered (e.g., NOT testing POLE in Stage IA Grade 1/2 endometrioid ECs who are usually untreated and stage III/IV ECs where ESMO/ESTRO/ESP recommendations are currently unchanged for POLEmut ECs) total costs saved increases to $\$ 720,294-\$ 958,842$ or $\$ 725-\$ 965 /$ capita. If costs of additional treatment directed by ProMisE are added, such as chemotherapy +/or radiation directed secondary to unveiling high risk molecular subtype, this would be expected to improve outcomes but at an additional cost of $\$ 280,908-\$ 826,719$ or \$282-\$832/capita.

Conclusions Molecular classification may tailor treatment with cost savings, while providing an opportunity to improve outcomes at reasonable cost for women with endometrial carcinoma. 